\title{
Terahertz Generation from Plasmonic Metasurfaces
}

\author{
Ming Fang, Zhixiang Huang, Member, IEEE, and \\ Xianliang $\mathrm{Wu}$ \\ Key Laboratory of Intelligent Computing and Signal \\ Processing, Ministry of Education, Anhui University, \\ Hefei, China (zxhuang@ahu.edu.cn)
}

\begin{abstract}
We numerically report a novel $\mathrm{THz}$ generation method based on plasmonic metasurface consist of single layer split-ring resonators array. The terahertz generation originates from nonlinear optical rectification in meta-atoms is broadband and free from phase match conditions. The strong linear response and inherent nonlinear behaviors in plasmonic metasurface can be described by a time-domain hydrodynamic model, in which both surface and bulk contributions of nonlinearity are considered. The coupled Maxwell-hydrodynamic system capturing full-wave physics and free electron dynamics is solved with a parallel finitedifference time-domain (FDTD) method. The proposed method is fundamentally important to design nonlinear plasmonic nanodevices, especially for efficient and broadband THz emitters.

Keywords-Hydrodynamic model, nonlinear plasmonic nanodevice, finite-difference time-domain (FDTD), terahertz emission, metasurface
\end{abstract}

\section{INTRODUCTION}

The field of plasmonic metamaterials has demonstrated spectacular experimental progress in recent years. One of the main driving forces in the field of metallic metamaterials is the potential applications of these materials in nanoantenna, nanosensing, and plasmonic devices [1,2]. In a recent work, by using difference-frequency generation, a terahertz $(\mathrm{THz})$ emitter based on a nonlinear metasurface was reported [3]. Efficient, broadband and tunable $\mathrm{THz}$ emission can be generated in a thin layer of metamaterial with tens of nanometers thickness by an infrared laser pumping. The $\mathrm{THz}$ emission intensity from the metamaterial is on the same order as the nonlinear crystals, and the ultra-thin $\mathrm{THz}$ emitter is free from quasi-phase-matching. However, due to the limitations of $\mathrm{THz}$ detectors, in particular, the upper cut-off frequency of the detectors between 2 and 4 $\mathrm{THz}$, the measured $\mathrm{THz}$ spectral bandwidths are narrower than the expectations of the nonlinear theory. To reveal the mechanism of $\mathrm{THz}$ generation from the metasurface, a timedomain implementation of the hydrodynamic model for electron dynamics in metals is developed. Both linear and nonlinear dynamics of the electrons in metamaterials are fully considered. The rigorous method allows us to study the $\mathrm{THz}$ generation from the plasmonic metamaterials and does not rely on the experimentally measured bulk and surface nonlinear susceptibilities. The proposed model can also be potentially used for investigating other quantum effects.

\author{
Wei E. I. Sha, Senior Member, IEEE \\ College of Information Science and Electronic Engineering \\ Zhejiang University, Huangzhou, China \\ (weisha@zju.edu.cn)
}

\section{METHOD}

The interaction of electromagnetic fields $\mathbf{E}$ and $\mathbf{H}$ with an arbitrary nonmagnetic (metallic) material can be described by

$$
\begin{array}{r}
\nabla \times \mathbf{H}=\varepsilon_{0} \frac{\partial \mathbf{E}}{\partial t}+\frac{\partial \mathbf{P}}{\partial t} \\
\nabla \times \mathbf{E}=-\mu_{0} \frac{\partial \mathbf{H}}{\partial t}
\end{array}
$$

where $\varepsilon_{0}$ and $\mu_{0}$ are the vacuum permittivity and permeability, and $\mathbf{P}$ is the polarization. To investigate the dynamics of electrons in a metallic/plasmonic system, the hydrodynamic model [3] is adopted

$$
\begin{gathered}
\frac{\partial \mathbf{v}}{\partial t}+\mathbf{v} \cdot \nabla \mathbf{v}=-\frac{e}{m}\left(\mathbf{E}+\mu_{0} \mathbf{v} \times \mathbf{H}\right)-\gamma \mathbf{v}-\frac{\nabla p}{n} \\
\frac{\partial n}{\partial t}=-\nabla \cdot(n \mathbf{v}),
\end{gathered}
$$

where $n(\mathbf{r}, t)$ and $\mathbf{v}(\mathbf{r}, t)$ are the time- and position-dependent electron density and velocity, $\gamma$ is the phenomenological damping frequency (capturing optical loss), $e$ and $m$ are the electron charge and mass, respectively, and $p$ is the quantum pressure $p=\left(3 \pi^{2}\right)^{2 / 3}(\hbar / 5) n^{5 / 3}$. The nonlinear terms $\mathbf{v} \cdot \nabla$ $\mathbf{v}, \mathbf{v} \times \mathbf{H}$, and $n \mathbf{v}$ underlie the physical origins of the nonlinear and nonlocal effects. Maxwell's equations (1-2) and hydrodynamic equations (3-4) are coupled through the polarization term $\frac{\partial \mathbf{P}}{\partial t}=-e n \mathbf{v}$. Equations (1) - (4) provide a self-consistent formulation of a free electron gas in plasmonic systems. Both nonlocal and nonlinear effects can be considered using the self-consistent formulations. The multiphysics equations can be numerically solved by employing the FDTD method [4-7].

\section{RESULTS}

An efficient and broadband $\mathrm{THz}$ emitter based on plasmonic metasurface is shown in Fig. 1 (a). The gold SRRs are arranged periodically in both $x$ and $y$ directions and supported by a layer of glass substrate coated with ITO. The thickness of the gold film, ITO and $\mathrm{SiO} 2$ substrates are $40 \mathrm{~nm}, 6 \mathrm{~nm}$ and $200 \mathrm{~nm}$, respectively. The parameters for gold are taken to be $n_{0}=$ 
$5.92 \times 10^{28} \mathrm{~m}^{-3}, \gamma=10.68 \times 10^{13} \mathrm{rad} / \mathrm{s}$. The dimensions of the SRRs were chosen as shown in Fig. 1 (b). for which the magnetic dipole can be excited around $195 \mathrm{THz}$ frequency. PMLs were set to be in the $z$ direction and periodic boundary conditions are employed at both the $x$ and $y$ directions. The results were calculated by adopting uniform spatial steps $\Delta x=\Delta y=\Delta z=2$ $\mathrm{nm}$, and a time step $\Delta t=3 \times 10^{-18} \mathrm{~s}$. A computational domain of $190 \times 190 \times 1000$ grids is used.

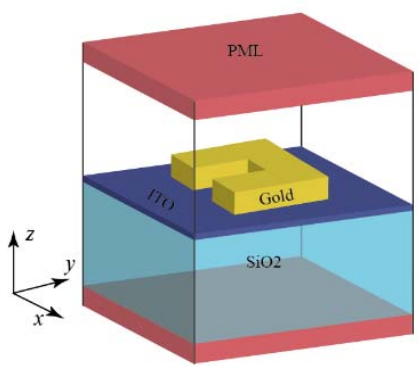

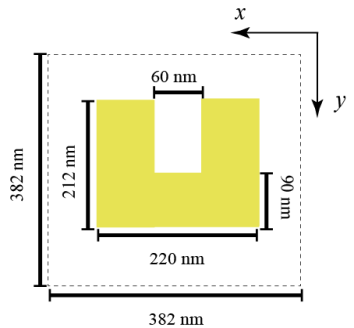

(b)

Fig. 1. (a) Layout of a metasurface consist of a periodic array of SRRs. (b) The feature size of a unit cell.

A Gaussian pulse laser $E(t)=E_{0} e^{-\left(t-t_{0}\right)^{2} / \tau^{2}} \cos \left(\omega_{0} t\right)$ is used as the pump source. The driving frequency, temporal width, change rate and peak amplitude of the pump laser are chosen as $\omega_{0}=1.228 \times 10^{15} \mathrm{rad} / \mathrm{s}$, and $E_{0}=2 \times 10^{7} \mathrm{~V} / \mathrm{m}$, respectively. Here $\tau=$ $150 \mathrm{fs}$ is the duration of excitation pulse corresponding to a spectral width $\sim 3 \mathrm{THz}$. Figure 2 shows the temporal profile of linear (a) and nonlinear transmitted field (b). The linear and nonlinear spectra are plotted in Fig. 2 (c) after the Fourier transform. We can see that the second-harmonic and differencefrequency generation are observed clearly. Figure 2 (d) is the $\mathrm{THz}$ signal in time domain. Note that the $\mathrm{THz}$ emission bandwidth of our results is broader than the observed $\mathrm{THz}$ spectrum in the experiment. This is because the upper cut-off frequency of the THz signal detector in the experiment is smaller than the theoretical prediction.

(a)

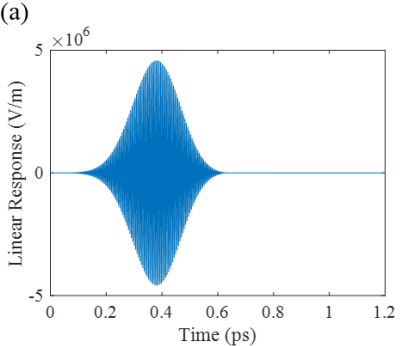

(c)

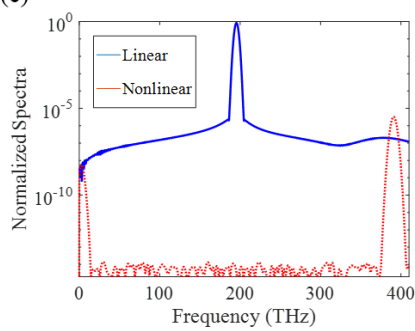

(b)

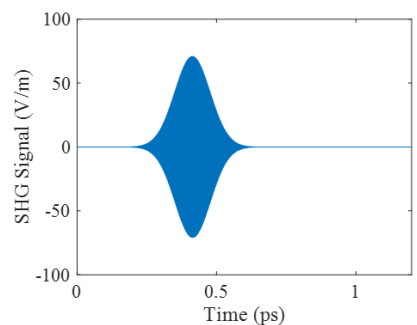

(c)

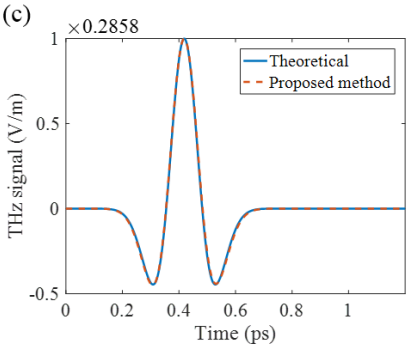

Fig. 2. The electric field as a function of time for the linear (a) and nonlinear (b) transmission. (c) Semi-log plot of the Fourier transform of the linear and nonlinear transmitted field versus frequency. (d) the normalized time-domain THz pulse.

\section{CONCLUSIONS}

In conclusion, we numerically demonstrate that The metasurface $\mathrm{THz}$ emitter can generate broadband $\mathrm{THz}$ signals. The results also show that the $\mathrm{THz}$ generation scheme is free from phonon limitations and quasi-phase-matching conditions. A self-consistent, accurate, and explicit FDTD based Maxwellhydrodynamic model has been developed for investigating and modeling both linear and nonlinear electromagnetic responses in plasmonic metasurfaces. Consequently, the proposed multiphysics model and FDTD scheme open unique opportunities for understanding and designing nonlinear plasmonic nanodevices.

\section{ACKNOWLEDGMENT}

This work was supported the NSFC (Nos. 61101064, 51277001 and 61201122), Universities Natural Science Foundation of Anhui Province (No. KJ2012A013), DFMEC (No. 20123401110009) and NCET (NCET-12-0596) of China.

\section{REFERENCES}

[1] C. M. Soukoulis and M. Wegener, "Past achievements and future challenges in the development of three-dimensional photonic metamaterials," Nature Photonics, vol. 5, no. 9, pp. 523-530, 2011.

[2] L. Novotny and N. van Hulst, "Antennas for light," Nature Photonics, vol. 5 , no. 2, pp. 83-90, 2011.

[3] L. Luo et al., "Broadband terahertz generation from metamaterials," Nature Communications, vol. 5, 2014.

[4] K. S. Yee, "Numerical Solution of Initial Boundary Value Problems Involving Maxwell's Equations in Isotropic Media," IEEE Trans. Antennas Propag. Vol. 14, no. 3, pp. 302-307, 1966.

[5] P. Ginzburg, A. V. Krasavin, G. A. Wurtz, and A. V. Zayats, "Nonperturbative Hydrodynamic Model for Multiple Harmonics Generation in Metallic Nanostructures," Acs Photonics, vol. 2, no. 1, pp. 8-13, 2015.

[6] C. Ciraci, E. Poutrina, M. Scalora, and D. R. Smith, "Origin of secondharmonic generation enhancement in optical split-ring resonators," Physical Review B, vol. 85, no. 20, 2012.

[7] M. Fang, Z. Huang, W. E. I. Sha, X. Y. Z. Xiong, and X. Wu, "Full Hydrodynamic Model of Nonlinear Electromagnetic Response in Metallic Metamaterials," Prog. Electromagn. Res. Vol. 157, pp. 63-78 2016 